\title{
The liver and muscle secreted Hfe2-protein maintains blood brain barrier integrity
}

\section{Xue Fan Wang}

University Health Network

\section{Robin Vigouroux}

Institut de la Vision https://orcid.org/0000-0002-3217-895X

\section{Yuriy Baglaenko}

University Health Network

\section{Angeliki Nikolakopoulou}

University of Southern California

\section{Dene Ringuette}

Krembil Research Institute

Horea Rus

University of Maryland

\section{Peter DiStefano}

University Health Network

\section{Suzie Dufour}

University Health network https://orcid.org/0000-0002-8439-4240

\section{Alireza Shabanzadeh}

Krembil Research Institute

\section{Michal Syonov}

Krembil Research Institute

\section{Seunggi Lee}

University Health Network

\section{Bernhard Mueller}

$$
\text { AbbVie }
$$

\section{Jason Charish}

Krembil Research Institute

\section{Hidekiyo Harada}

Krembil Research Institute

\section{Jason Fish}

https://orcid.org/0000-0003-0640-7277

Joan Wither

University Health Network

Thomas Wälchli 
Krembil Research Institute

Jean-Francois Cloutier

Montreal Neurological Institute

\section{Berislav Zlokovic}

University of Southern California https://orcid.org/0000-0002-6802-8232

\section{Peter Carlen}

Krembil Research Institute https://orcid.org/0000-0002-3339-8102

\section{Philippe Monnier ( $\square$ pmonnier@uhnres.utoronto.ca )}

University Health Network https://orcid.org/0000-0002-6221-4130

\section{Article}

\section{Keywords:}

Posted Date: December 2nd, 2021

DOl: https://doi.org/10.21203/rs.3.rs-1028867/v1

License: (c) (i) This work is licensed under a Creative Commons Attribution 4.0 International License. Read Full License 


\section{Abstract}

Liver failure causes blood-brain-barrier (BBB) breakdown leading to central nervous system damage, however the mechanisms whereby the liver influences BBB-integrity remain elusive. One possibility is that the liver secretes an as-yet to be identified molecule(s) that circulate in the serum to directly promote BBB integrity. We developed light-sheet imaging for three-dimensional study of BBB function. We show that liver- or muscle-specific knockout of Hfe2 induces BBB breakdown, leading to accumulation of toxicblood-derived fibrinogen in the brain, lower cortical neuron numbers, and behavioral deficits. In healthy animals, soluble Hfe2 competes with its homologue RGMa for binding to Neogenin, thereby blocking RGMa-induced downregulation of PDGF-B and Claudin-5 in endothelial cells and the ensuing BBB disruption. Hfe2 administration in an animal model of multiple sclerosis prevented paralysis and immune cell infiltration by inhibiting RGMa-mediated BBB alteration. This study has implications for the pathogenesis and potential treatment of diseases associated with BBB dysfunction such as multiple sclerosis.

\section{One Sentence Summary}

Serum levels of RGMa and Hfe2 regulate BBB-integrity, which has direct implications for many neurological conditions including MS.

\section{Main Text}

The association between liver disease and behavioral disturbances was recognized by the father of Western medicine, Hippocrates (460-371 B.C.). Clinical observations have documented that liver diseases are associated with alterations in the Central Nervous System (CNS) that manifest as behavioral changes such as cognitive dysfunction, mood disorders and sleep disturbance ${ }^{1}$. Although these behavioral alterations greatly impact quality of life, the pathways connecting the liver and the brain, which mediate CNS-conditions, remain elusive.

The CNS is protected by the blood-brain-barrier (BBB), which is formed by endothelial cells, astrocyte endfeet, and pericytes. Alterations of the BBB is a common theme in many CNS conditions including multiple sclerosis (MS), Alzheimer's, and stroke ${ }^{2}$, and may expose the brain parenchyma to harmful substances from the circulating blood ${ }^{3}$. Blood born proteins, such as fibrinogen, have detrimental effects on neurons, which may account for most of the behavioral deficits observed in these pathologies ${ }^{3}$. BBB-disruption has been observed in several liver pathologies, such as liver failure, resulting in brain edema ${ }^{4}$. The mechanisms that link liver function and BBB-integrity are largely unknown. One possibility is that the diseased liver secretes factors such as matrix metalloproteases that are detrimental to BBB-integrity ${ }^{4}$. Another unexplored possibility is that the healthy liver secretes one or several factors that serve to maintain BBB function. 
Repulsive Guidance Molecules (RGMs) are GPI-anchored proteins comprising 3 members: RGMa, RGMb and $\mathrm{RGMc} / \mathrm{Hfe} 2 / \mathrm{Hjv}^{5}$. They exert their biological functions through interaction with the transmembrane receptor Neogenin and shared co-receptors, the Bone Morphogenic Proteins (BMPs) ${ }^{6}$. Hfe2 is mainly produced by the liver, where it regulates iron homeostasis, and by the muscles, where it is involved in skeletal muscle differentiation ${ }^{7}$. RGMa and RGMb are expressed in many organs, but studies have mostly focused on their role in the developing and diseased CNS ${ }^{8}$. In the diseased CNS, RGMa blocks axonal regeneration and modulates neuronal death 9,10 . A potential involvement of RGMa in MS was first proposed from a genetic study showing that a single nucleotide polymorphism in RGMa was associated with MS and was positively correlated with changes in the expression of inflammatory cytokines in the cerebrospinal fluid of MS patients ${ }^{11}$. Other studies showed that inhibiting the RGMa-Neogenin pathway abolishes the $T$ cell response in experimental autoimmune encephalitis (EAE), which ameliorates clinical symptoms ${ }^{12}$. Furthermore, RGMa neutralization promotes axon regeneration and functional recovery in animals with $E A E^{13}$. We sought to investigate the role of RGM-proteins on BBB integrity and its role in disease models.

\section{Results}

\section{Hfe2 liver knock out alters BBB integrity}

Using widefield imaging, we observed that $\mathrm{Hfe} 2$ knock-out mice demonstrated an intensive leakage pattern, indicative of BBB breakdown (Extended Data Fig. 1a-c). This was unexpected as $\mathrm{Hfe} 2$ is not expressed in the brain ${ }^{5}$ (Extended Data Fig. 1d) and suggested that a non-brain source of Hfe2 regulates $\mathrm{BBB}$ integrity. $\mathrm{Hfe} 2$ is mainly expressed by the liver ${ }^{14,15}$; hence we hypothesized that $\mathrm{Hfe} 2$ released by the liver into the systemic blood stream regulates BBB integrity. To investigate the role of liver-secreted $\mathrm{Hfe} 2$ on BBB integrity, we generated $H f e 2^{\Delta A l b-c r e}\left(H f e 2^{f / f l} ; A l b-c r e\right)$ transgenic mice to genetically ablate Hfe2 production, specifically in the liver. ELISA analysis of the serum demonstrated a $56 \pm 8 \%$ reduction of Hfe2 levels in the serum, confirming that the liver is a major source of serum Hfe2 (Extended Data Fig. 1e).

To characterize BBB integrity in detail, we developed a method allowing for 3D representations of BBBleakages at any given cortical depth (Fig. 1a). Leakages were defined by the extravasation of Lysinefixable Texas Red-conjugated $70 \mathrm{kDa}$ dextran (TR-dextran). Animals were perfused to remove residual TRdextran in the vessels. By using CUBIC lipid-clearing, the optically transparent brains were imaged using light-sheet microscopy to map out BBB-leakage throughout the whole brain. In order to ensure that our perfusion protocol removed all traces of TR-dextran in the intact brain, while also allowing for visualization of BBB-leakages, we performed middle cerebral artery occlusion (MCAO), which is known to open the BBB of one half of the brain while leaving the other half unperturbed. As expected, leakagestaining was seen in the MCAO damaged hemisphere, while the healthy hemisphere remained devoid of any leakage staining (Extended Data Fig. 2). Having validated our perfusion protocol, we studied the role of liver $\mathrm{Hfe} 2$ on BBB integrity. In $H f e 2^{\triangle A l b-c r e}$ animals, we observed intense BBB-leakage in superficial as 
well as deep regions of the brain, whereas $H f e 2^{f / f l}$ control littermates did not display any such leakage (Fig. 1b).

To ensure that the BBB-leakage observed in the $H f e 2^{\triangle A l b-c r e}$ animals did not result from a developmental defect, we ablated $\mathrm{Hfe} 2$ secretion specifically in adult mice. We infected a cohort of adult $H f e 2^{\text {fl/fl }}$ mice with a liver-directed adenovirus, AAV8-AlbCre ${ }^{16}$, or a control adenovirus, AAV8-GFP. Three weeks postinfection, AAV8-AlbCre infected mice displayed significant reduction in $\mathrm{Hfe} 2$ serum-levels, which was comparable to that of $\mathrm{Hfe} 2^{\triangle \mathrm{Alb}-\mathrm{cre}}$ mice (Extended Data Fig. $1 \mathrm{e}$ ). $\mathrm{Hfe} \mathrm{2}^{\mathrm{f} / \mathrm{fl}}$ mice infected with AAV8-AlbCre invariably displayed severe BBB-leakages, which were not observed in the AAV8-GFP infected controls (Fig. 1b). Both Hfe2 liver-specific knockout models exhibited significant BBB-leakage, as indicated by increased total fluorescence intensity of residual TR-dextran, indicating that $\mathrm{Hfe} 2$ plays a significant role on maintaining BBB integrity (Fig. 1b). To confirm this phenotype and validate our BBB-assessment method, we performed in-vivo multiphoton imaging of TR-dextran in $\mathrm{Hfe}^{\triangle \mathrm{Allb}-\mathrm{cre}}$ and AAV8-AlbCre mice along with their respective controls. We confirmed that the observed leakage was occurring within the brain, by imaging the height of the dura relative to the observed blood vessels (Supplementary Video 1). Following this validation, we did time-lapse imaging over a 40-minute window to record the temporal dynamics of the leakage. As expected, both $\mathrm{Hfe} 2$ liver knock-out mouse models displayed obvious extravascular leakage (Fig. 1c and Supplementary Video 2). Time-series analysis revealed a significant increase in the total TR-dextran extravasation in the knockout animals compared to their respective controls (Fig. 1C) and this extravasation was observed only in larger blood vessels (>24 $\mu \mathrm{m}$, Extended Data Fig. 1f). The leakage pattern seen in the in-vivo imaging supported what was observed in our lightsheet BBB assessments (Fig. 1b). We subsequently investigated whether BBB-breakdown in Hfe2deficient mice led to accumulation of blood-born substances in the CNS. We performed staining for blood-born Fibrinogen, a well-established marker of BBB-breakdown ${ }^{17}$. Immunostaining for fibrinogen and the endothelial marker isolectin revealed abundant fibrinogen deposition around the cerebral vasculature in $\mathrm{Hfe}$-deficient mice, which was significantly elevated compared to controls (Fig. 1d). Finally, we performed intravenous injection of TR-dextran and studied TR-dextran presence in sections (Fig. 1e and Extended Data Fig. 1g) ${ }^{18}$. We observed TR-dextran deposition similar to that observed by others demonstrating BBB disruption in mice ${ }^{18}$. In conclusion, using 4 different experimental techniques we demonstrate that levels of $\mathrm{Hfe} 2$ are a critical regulator of BBB-integrity.

\section{Hfe2 muscle knock out alters BBB integrity}

$\mathrm{Hfe} 2$ plays a major role in iron homeostasis, and $H f 2^{\Delta \mathrm{Alb}-\mathrm{cre}}$ mice display a $52 \pm 13 \%$ increase in iron levels in the blood (Extended Data Fig. 3a) ${ }^{15}$. To test if altered iron levels were the cause of the BBBleakage, we generated $H f e 2^{\Delta A c t a-c r e}\left(H f e 2^{f / f l} ;\right.$ Acta-cre) transgenic mice to genetically ablate $\mathrm{Hfe} 2$ production specifically in skeletal muscles. Analysis demonstrated that this procedure reduced $\mathrm{Hfe} 2$ levels by $32 \pm 10 \%$ without altering iron levels in the serum (Extended Data Fig. 3a-b) ${ }^{15}$. In Hfe ${ }^{\triangle A c t a c r e}$ animals, we nonetheless observed BBB-leakages using light sheet imaging and in-vivo multiphoton imaging, suggesting BBB-alteration is not the result of increased of iron levels (Fig. $1 \mathrm{f}$ and Extended Data 
Fig. 1h). Leakages appeared less pronounced than in $H f e 2^{\Delta a l b-c r e}$ animals, which may reflect the lower decrease in blood $\mathrm{Hfe} 2$ in $\mathrm{Hfe}^{\Delta \mathrm{Acta}-c r e}$ when compared to $\mathrm{Hfe}^{\mathrm{\Delta alb}-\mathrm{cre}}$. Although we cannot totally exclude a role for iron in the leakage observed in $\mathrm{Hfe}^{\mathrm{\Delta alb}-\mathrm{cre}}$ animals, these results indicate that liver-and muscle-secreted Hfe2 may play a pivotal role in the maintenance of BBB-integrity.

\section{Hfe2 muscle-and liver-knock out results in neuronal loss and behavioral deficits}

BBB disruption in $H f e 2^{\triangle A l b-c r e}$ animals triggers the extravasation of fibrinogen, which can be toxic to brain neurons ${ }^{19}$, hence, we surveyed the number of cortical neurons in $\mathrm{Hfe}^{\triangle \mathrm{Alb}-\mathrm{cre}}, \mathrm{Hfe} 2^{\triangle \mathrm{Acta}-\mathrm{cre}}$, and AAV8-AlbCre animals. Interestingly, in both the liver- and muscle-specific $\mathrm{Hfe} 2 \mathrm{knock}$ outs, we observed a strong reduction of the number of cortical neurons (Fig. 2a). The observed reduction in the number of neurons in $\mathrm{Hfe} 2^{\triangle A c t a-c r e}$ animals fits data showing that a serum pool of $\mathrm{Hfe} 2$ serves to regulate $B B B$ integrity. Following infection with AAV8-AlbCre, to ablate liver production of $\mathrm{Hfe} 2$, we also observed a progressive loss of cortical neurons over 3 to 4 weeks (Fig. 2a). Next, we performed a series of behavioral tests to determine whether neuronal loss correlated with functional deficits. Hfe2 knock out in the liver resulted in a strong reduction of functional scores in marble burying, open field, and water maze tests, indicating impaired brain functions (Fig. 2b-d). Similarly, Hfe $2^{\Delta \text { Acta-cre }}$ animals also displayed functional deficits, however in this case we cannot exclude the possibility that this is a result of muscle weakness.

\section{RGMa and Hfe2 have opposite effects on Claudin-5 and PDGF-B expression}

Pericytes are key to maintaining BBB-integrity ${ }^{20,21}$, hence we studied pericyte coverage of endothelial cells in Hfe2 liver KO (Fig. 3a). Interestingly, both $\mathrm{Hfe}^{\mathrm{\Delta alb} \text {-cre }}$ and AAV8-AlbCre animals displayed a significant reduction of pericyte coverage when compared to control animals, suggesting that this alteration of pericyte coverage may trigger BBB-breakdown (Fig. 3a). PDGF-B expression by endothelial cells is critical for maintaining pericyte coverage. Consequently, we used quantitative PCR on purified endothelial cells to determine whether knocking out Hfe2 affects mRNA levels for PDGF-B (Fig. 3b). PDGF-B mRNA levels were reduced by $\sim 4.5 \pm 1.6 \times$ fold in $H f e 2^{\Delta A l b-c r e}$ when compared to $H f e 2^{f / / f l}$ mice (Fig. $3 b$ ), suggesting that $\mathrm{Hfe} 2$ contributes to maintenance of BBB integrity by promoting PDGF-B expression. To investigate the mechanisms whereby $\mathrm{Hfe} 2$ regulates PDGF-B expression, we studied PDGF-B mRNA levels in cultured endothelial cells. Surprisingly, addition of $\mathrm{Hfe} 2$ to endothelial cultures did not influence PDGF-B mRNA levels (Fig. 3c). We hypothesized that $\mathrm{Hfe} 2$ may counteract the effect of another factor that negatively regulates PDGF-B expression in endothelial cells (bEnd3 cells). In axonal growth experiments, we have observed that $\mathrm{Hfe} 2$ blocks the inhibitory activity of RGMa. The addition of RGMa to the laminin-substrate shortens axons when compared to laminin on its own, while the addition of $\mathrm{Hfe} 2$ to the medium restored axonal length to control levels, suggesting that $\mathrm{Hfe} 2$ can fully neutralize RGMa (Extended Data Fig. 4). We detected RGMa in human and murine blood serum by Western Blotting 
(Extended Data Fig. 5) and ELISA ( 0.9 $\mu \mathrm{g} / \mathrm{mL})$, respectively, indicating that endothelial cells are in direct contact with both blood-borne RGMa and Hfe2. We addressed the possibility that RGMa and Hfe2 similar to what was observed in growing axons - have opposite effect on endothelial cells. RGMa was added to the medium of cultured bEnd 3 cells. This induced a $4.1 \pm 1.3 \times$ fold reduction in PDGF-B mRNA levels, which prompted us to test whether Hfe2 positively impacts PDGF-B expression by counteracting this RGMa effect (Fig. 3c). As predicted, when Hfe2 was added to RGMa treated cells, we observed an abrogation of the RGMa effect on PDGF-B mRNA levels (Fig. 3c). PDGF-B is present in the matrix of endothelial cells, hence, we evaluated the expression of this protein using immunohistochemistry. In agreement with our mRNA data, RGMa addition to the medium reduced PDGF-B, which was rescued by the addition of Hfe2 to the medium (Extended Data Fig. 6).

BBB integrity is also regulated by tight junctional molecules, hence, we asked whether the expression of tight junction proteins is altered upon ligand treatment in the cultured bEnd3 cells. Immunocytochemistry revealed obvious claudin-5 discontinuity upon RGMa treatment, and such disruption was prevented by Hfe2 co-treatment (Fig. 3d). Western blot further confirmed a significant reduction in claudin-5 expression in RGMa-treated bEnd3 cells compared to Hfe2 co-treatment and controls (Fig. 3e). RGMa did not appear to impact the expression of Occludin, another tight junction protein (not shown). Hence our results indicated that RGMa treatment on cerebral endothelial cells significantly alters both the expression of Claudin-5 and PDGF-B, which can be completely prevented by Hfe2.

\section{RGMa and Hfe2 have opposite effects on endothelial cells and BBB integrity}

To further assess the effect of RGMa and Hfe2 on BBB integrity, we performed a Transwell permeability assay to study the extravasation of horse radish peroxidase (HRP) through a monolayer of bEnd 3 cells upon ligand treatment. We found that soluble RGMa significantly increases the monolayer permeability to HRP to an extent comparable with TNF-a, which was used as a positive control. The effect of RGMa on the bEnd3 monolayer was significantly attenuated by the addition of $\mathrm{Hfe} 2$ (Fig. 3f). Additional in-vitro assessment using trans-endothelial electrical resistance (TEER) confirmed that RGMa significantly reduced bEnd3 barrier function which was suppressed upon $\mathrm{Hfe} 2$ co-treatment (Fig. 3g).

The above presented data suggest that RGMa alters endothelial barrier function in vitro. To address this in vivo, we intravenously administered either soluble RGMa alone or with $\mathrm{Hfe} 2$ into wild type (WT) mice and assessed BBB-integrity $24 \mathrm{~h}$ post-injection. Using the light-sheet BBB assessment tool, we observed intense accumulation of TR-dextran in the brains of RGMa-treated WT mice, indicating that RGMainjection severely disrupts BBB function (Fig. 4a). Co-treatment of RGMa and Hfe2 significantly prevented RGMa-mediated BBB-breakdown (Fig. 4a and Extended Data Fig. 7). Multiphoton imaging further confirmed the disruptive role of RGMa, which displayed a significant increase in TR-dextran extravasation. BBB-integrity in WT animals co-treated with both RGMa and Hfe2 were comparable to the PBS treatment (Fig. 4b and Supplementary Video 3). RGMa-treated WT animals exhibited a significant amount of perivascular fibrinogen accumulation, which was completely abrogated by $\mathrm{Hfe} 2$ co-treatment 
(Fig. 4c). Furthermore, we looked at BBB-leakage using widefield imaging, confirming that RGMa induces leakage that is not observed when Hfe2 is co-injected (Extended Data Fig. 7). Together, these data identify RGMa and Hfe2 as novel regulators of PDGF-B and BBB integrity.

\section{Neogenin is involved in RGMa mediated opening of the BBB}

RGMa interacts with Neogenin to inhibit axonal growth ${ }^{22}$. Although $\mathrm{Hfe} 2$ also interacts with Neogenin, $\mathrm{Hfe} 2$ does not inhibit outgrowth, but rather suppresses the RGMa inhibition of axonal growth (Extended Data Fig. 4). In the chick brain, Hfe2 did not lead to a displacement of the Neogenin receptor and it still localized to lipid rafts (data not shown), hence, these outgrowth data suggest that Hfe2 prevents the interaction between RGMa and Neogenin. To address this possible role for $\mathrm{Hfe} 2$, we developed an assay in which soluble Neogenin (AP-tagged) will interact with RGMa. We observed that soluble Neogenin-AP interacts with ELISA-plate coated RGMa (Fig. 5a). In a competitive binding assay, we show that Hfe2 significantly prevented the binding of Neogenin-AP to RGMa (Fig. 5a). Also, we show that Neogenin-AP binds to $\mathrm{Hfe} 2$ and that this interaction is blocked by RGMa (Fig. 5a). Next, we studied Neogenin expression in blood vessels, and show that it is strongly expressed in endothelial cells (Fig. 5b). Neogenin staining was observed in the lumen of human and murine blood vessels suggesting that it interacts with blood proteins (Fig. 5b). The presence of Neogenin in endothelial cells raised the possibility that Hfe2 restores BBB integrity by preventing RGMa binding to Neogenin. A hypomorphic allele with $~ 90 \%$ loss of Neogenin has been described previously, and shows that mice die around P28 ${ }^{23}$. Hence, to study the involvement of Neogenin in RGMa-induced BBB breakdown, we used $\mathrm{Neo}^{\Delta \mathrm{Tie} 2-\mathrm{creERT2}}\left(\mathrm{Neo}{ }^{\mathrm{fl} / \mathrm{fl}}\right.$; Tie2creERT2) knockout animals in vivo, wherein Neogenin is genetically deleted from endothelial cells upon Tamoxifen induction. Immunoblotting on isolated cerebral endothelial cells of $N e o^{\Delta T i e 2-c r e E R T 2}$ revealed complete deletion of Neogenin (Extended Data Fig. 8). Soluble RGMa treatment applied to tamoxifentreated $\mathrm{NeO}^{\triangle \mathrm{Ti} \text { 2-creERT2 }}$ mice did not induce BBB-leakage as observed with WT mice, indicating that RGMa-mediated BBB breakdown is primarily mediated by Neogenin (Fig. $5 \mathrm{c}$ and Extended Data Fig. 8). Multiphoton imaging as well as fibrinogen staining in RGMa-treated $\mathrm{NeO}^{\Delta \mathrm{Tie} 2-\mathrm{creERT} 2}$ mice also indicated that Neogenin ablation in cerebral endothelial cells significantly prevents RGMa-mediated BBB alterations (Fig. 5d-f and Supplementary Video 4). These results demonstrate the role of the Neogenin receptor in mediating the effect of RGMa on BBB integrity.

\section{The RGM/Neogenin pathway modulates BBB integrity in a model of MS}

RGMa is upregulated in MS, a disease manifesting severe BBB-disruption ${ }^{12,13}$. Furthermore, inflammation has been shown to reduce Hfe2/RGMc expression ${ }^{24}$. Therefore, we explored the possibility that the BBB-breakdown caused by RGMa/Hfe2 imbalance may be a critical component in MS pathology. To test this hypothesis, we used EAE, an animal model for MS ${ }^{12}$. Animals received a MOG peptide that induces paralysis within 2-3 weeks. ELISA and Western Blotting showed that Hfe2-levels are strongly downregulated in the serum of EAE mice when compared to sham induction mice (Fig. 6a). Interestingly RGMa levels were increased in EAE animals (Fig. 6a) and we observed high levels of RGMa around blood 
vessels within the plaques found in the brain of MS patients (Extended Data Fig. 9). We reasoned that low $\mathrm{Hfe} 2$ combined with high RGMa levels open the BBB in EAE and that administrating $\mathrm{Hfe} 2$ should restore the RGMa/Hfe2 balance and prevent RGMa-mediated BBB opening. Treatment with soluble Hfe2 to EAE mice significantly delayed disease onset and significantly reduced disease severity (Fig. 6b). This effect was suppressed by co-injecting RGMa with $\mathrm{Hfe2}$, which fits with the opposite activities of RGMa and $\mathrm{Hfe} 2$ shown earlier (Fig. 3\&4). Because Hfe2-liver knock out reduces $\mathrm{Hfe} 2$ levels in the serum and alters BBB-integrity, $\mathrm{Hfe}^{\mathrm{AAlb}-\mathrm{cre}}$ mice should present an aggravation of EAE symptoms. Indeed, $\mathrm{Hfe} 2^{\Delta \mathrm{Alb}-\mathrm{cre}}$ mice displayed earlier disease onset and significantly increased disease severity at early stages of the disease when compared to controls (Fig. 6c). These data suggest that circulating Hfe2 prevents functional impairment by blocking the detrimental effects of RGMa following EAE induction. Because Neogenin mediates the RGMa effect on BBB (Fig. 5), we tested whether Neogenin knock-out in endothelial cells prevents EAE symptoms. As expected, $\mathrm{Neo}^{\Delta T \mathrm{Tie} 2 \text {-creERT2 }}$ mice exhibited significant improvement following EAE induction (Extended Data Fig. 10), which fits with a model in which Neogenin mediates the RGMa/Hfe2 effect.

Examination of cell infiltrates within the spinal cord of the Hfe2-treated EAE mice showed significant reduction of cellular infiltrates as assessed using the H\&E staining inflammatory index, where 0 is no infiltrates and 4 is cellular infiltration within the grey and white matter (Extended Data Fig. 10). Immune cells play a critical role in CNS damage in $\mathrm{EAE}^{25}$, hence we examined immune cells infiltrations in spinal cord. Hfe2-treated mice showed significant reduction in the pan $\mathrm{T}$ cell marker CD3+, the pan B cell marker B220+, as well as the myeloid marker CD11B+ when compared to vehicle-treated controls (Fig. 6d). To ensure that reduced immune cell infiltration did not result from an effect of $\mathrm{Hfe} 2$ on immune cell activation, we performed adoptive transfer of activated immune cells into the recipient mice (Fig. 6e). $\mathrm{Hfe} 2$ treatment once again prevented the infiltration of immune cells into the CNS indicating that the positive effect of $\mathrm{Hfe} 2$ on EAE progression was likely independent of any effects on immune cell priming (Fig. 6e). Treatment with $\mathrm{Hfe} 2$ had no effect on i) the adhesion properties of naïve T and B cells, ii) naïve antigen presenting cells, iii) naïve immune cell populations, iv) on activated immune cells, and $v$ ) antigen specific immune cells which also indicates that the $\mathrm{Hfe} 2$ effect is independent of immune cell priming (Extended Data Fig. 11a-e). This suggests that $\mathrm{Hfe} 2$ prevents immune cell infiltration by preventing BBBalteration following EAE induction, which was further confirmed by a significant reduction in endogenous fibrinogen extravasation into the CNS of EAE mice treated with Hfe2 (Extended Data Fig. 11f). Taken together, our data validate a role for $\mathrm{Hfe} 2, \mathrm{RGMa}$, and Neogenin in BBB-maintenance and highlight their importance in MS pathology.

\section{Discussion}

Here, we demonstrate that the healthy liver contributes to BBB maintenance. Our surprising findings that a liver-secreted protein plays a key role in the maintenance of the highly specialized and tightly regulated CNS vasculature ${ }^{26-28}$ warrants further investigation. For instance, it remains to be shown whether any other liver protein is involved in BBB maintenance. The molecular interplay between $\mathrm{Hfe} 2$ and RGMa 
underlying BBB maintenance not only provides a novel therapeutic approach in numerous diseases manifesting severe BBB disruption, such as Alzheimer's disease ${ }^{29,30}$, stroke ${ }^{31}$, Parkinson's disease ${ }^{30}$, and brain tumors ${ }^{32,33}$, but also can be exploited as a switch for transient opening of BBB allowing the efficient delivery of treatment drugs into the CNS.

\section{Declarations}

\section{Acknowledgements}

We thank Mr. Marc Schwab for assistance with the endothelial cell isolation procedures. This work was supported by the Krembil Foundation, Heart and Stroke Foundation of Ontario (Grant number NA7067), The Canadian Foundation for Innovation and the Canadian Institutes for Health Research (Grant number PJT 162094).

\section{References}

1. D'Mello, C. \& Swain, M. G. Liver-brain inflammation axis. Am J Physiol Gastrointest Liver Physio/ 301, G749-761, doi:10.1152/ajpgi.00184.2011 (2011).

2. Piers, T. M. et al. Soluble Fibrinogen Triggers Non-cell Autonomous ER Stress-Mediated MicroglialInduced Neurotoxicity. Front Cell Neurosci 12, 404, doi:10.3389/fncel.2018.00404 (2018).

3. Petersen, M. A., Ryu, J. K. \& Akassoglou, K. Fibrinogen in neurological diseases: mechanisms, imaging and therapeutics. Nat Rev Neurosci 19, 283-301, doi:10.1038/nrn.2018.13 (2018).

4. Nguyen, J. H. et al. Matrix metalloproteinase-9 contributes to brain extravasation and edema in fulminant hepatic failure mice. J Hepatol 44, 1105-1114, doi:10.1016/j.jhep.2005.09.019 (2006).

5. Niederkofler, V., Salie, R., Sigrist, M. \& Arber, S. Repulsive guidance molecule (RGM) gene function is required for neural tube closure but not retinal topography in the mouse visual system. $J$ Neurosci 24 , 808-818, doi:10.1523/JNEUROSCI.4610-03.2004 (2004).

6. Siebold, C., Yamashita, T., Monnier, P. P., Mueller, B. K. \& Pasterkamp, R. J. RGMs: Structural Insights, Molecular Regulation, and Downstream Signaling. Trends Cell Biol 27, 365-378, doi:10.1016/j.tcb.2016.11.009 (2017).

7. Severyn, C. J. \& Rotwein, P. Conserved proximal promoter elements control repulsive guidance molecule c/hemojuvelin (Hfe2) gene transcription in skeletal muscle. Genomics 96, 342-351, doi:10.1016/j.ygeno.2010.09.001 (2010).

8. Monnier, P. P. et al. RGM is a repulsive guidance molecule for retinal axons. Nature 419, 392-395, doi:10.1038/nature01041 (2002).

9. Hata, K. et al. RGMa inhibition promotes axonal growth and recovery after spinal cord injury. $J$ Cell Biol 173, 47-58, doi:10.1083/jcb.200508143 (2006).

10. Mothe, A. J. et al. RGMa inhibition with human monoclonal antibodies promotes regeneration, plasticity and repair, and attenuates neuropathic pain after spinal cord injury. Sci Rep 7, 10529, 
doi:10.1038/s41598-017-10987-7 (2017).

11. Nohra, R. et al. RGMA and IL21R show association with experimental inflammation and multiple sclerosis. Genes Immun 11, 279-293, doi:10.1038/gene.2009.111 (2010).

12. Muramatsu, R. et al. RGMa modulates T cell responses and is involved in autoimmune encephalomyelitis. Nat Med 17, 488-494, doi:10.1038/nm.2321 (2011).

13. Demicheva, E. et al. Targeting repulsive guidance molecule $A$ to promote regeneration and neuroprotection in multiple sclerosis. Cell Rep 10, 1887-1898, doi:10.1016/j.celrep.2015.02.048 (2015).

14. Silvestri, L., Pagani, A. \& Camaschella, C. Furin-mediated release of soluble hemojuvelin: a new link between hypoxia and iron homeostasis. Blood 111, 924-931, doi:10.1182/blood-2007-07-100677 (2008).

15. Chen, W., Huang, F. W., de Renshaw, T. B. \& Andrews, N. C. Skeletal muscle hemojuvelin is dispensable for systemic iron homeostasis. Blood 117, 6319-6325, doi:10.1182/blood-2010-12327957 (2011).

16. Pradhan-Sundd, T. et al. Dual catenin loss in murine liver causes tight junctional deregulation and progressive intrahepatic cholestasis. Hepatology, doi:10.1002/hep.29585 (2017).

17. Nag, S., Manias, J. L., Kapadia, A. \& Stewart, D. J. Molecular Changes Associated with the Protective Effects of Angiopoietin-1 During Blood-Brain Barrier Breakdown Post-Injury. Mol Neurobio/ 54, 42324242, doi:10.1007/s12035-016-9973-4 (2017).

18. Armulik, A. et al. Pericytes regulate the blood-brain barrier. Nature 468, 557-561, doi:10.1038/nature09522 (2010).

19. Montagne, A. et al. Pericyte degeneration causes white matter dysfunction in the mouse central nervous system. Nat Med 24, 326-337, doi:10.1038/nm.4482 (2018).

20. Ben-Zvi, A. et al. Mfsd2a is critical for the formation and function of the blood-brain barrier. Nature 509, 507-511, doi:10.1038/nature13324 (2014).

21. Andrae, J., Gallini, R. \& Betsholtz, C. Role of platelet-derived growth factors in physiology and medicine. Genes Dev 22, 1276-1312, doi:10.1101/gad.1653708 (2008).

22. Rajagopalan, S. et al. Neogenin mediates the action of repulsive guidance molecule. Nat Cell Biol6, 756-762, doi:10.1038/ncb1156 (2004).

23. Lin, S. et al. Neogenin-loss in neural crest cells results in persistent hyperplastic primary vitreous formation. J Mol Cell Biol 12, 17-31, doi:10.1093/jmcb/mjz076 (2020).

24. Krijt, J., Vokurka, M., Chang, K. T. \& Necas, E. Expression of Rgmc, the murine ortholog of hemojuvelin gene, is modulated by development and inflammation, but not by iron status or erythropoietin. Blood 104, 4308-4310, doi:10.1182/blood-2004-06-2422 (2004).

25. Holz, K. et al. Differing Outcome of Experimental Autoimmune Encephalitis in Macrophage/Neutrophil- and T Cell-Specific gp130-Deficient Mice. Front Immuno/ 9, 836, doi:10.3389/fimmu.2018.00836 (2018). 
26. Walchli, T. et al. Wiring the Vascular Network with Neural Cues: A CNS Perspective. Neuron 87, $271-$ 296, doi:10.1016/j.neuron.2015.06.038 (2015).

27. Quaegebeur, A., Lange, C. \& Carmeliet, P. The neurovascular link in health and disease: molecular mechanisms and therapeutic implications. Neuron 71, 406-424, doi:10.1016/j.neuron.2011.07.013 (2011).

28. Walchli, T. et al. Quantitative assessment of angiogenesis, perfused blood vessels and endothelial tip cells in the postnatal mouse brain. Nat Protoc 10, 53-74, doi:10.1038/nprot.2015.002 (2015).

29. Montagne, A. et al. Blood-brain barrier breakdown in the aging human hippocampus. Neuron 85 , 296-302, doi:10.1016/j.neuron.2014.12.032 (2015).

30. Sweeney, M. D., Sagare, A. P. \& Zlokovic, B. V. Blood-brain barrier breakdown in Alzheimer disease and other neurodegenerative disorders. Nat Rev Neuro/ 14, 133-150, doi:10.1038/nrneurol.2017.188 (2018).

31. Yang, Y. \& Rosenberg, G. A. Blood-brain barrier breakdown in acute and chronic cerebrovascular disease. Stroke 42, 3323-3328, doi:10.1161/STROKEAHA.110.608257 (2011).

32. Aldape, K. et al. Challenges to curing primary brain tumours. Nat Rev Clin Oncol 16, 509-520, doi:10.1038/s41571-019-0177-5 (2019).

33. Arvanitis, C. D., Ferraro, G. B. \& Jain, R. K. The blood-brain barrier and blood-tumour barrier in brain tumours and metastases. Nat Rev Cancer 20, 26-41, doi:10.1038/s41568-019-0205-x (2020).

\section{Figures}



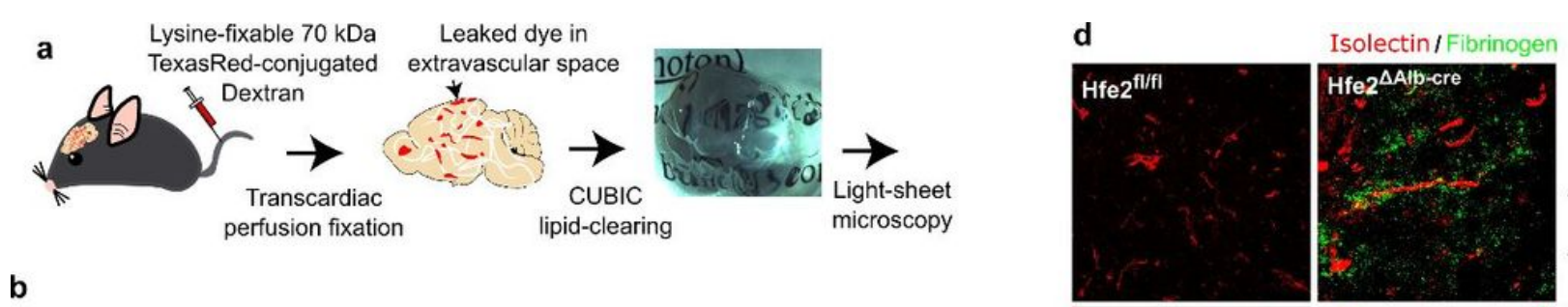

b
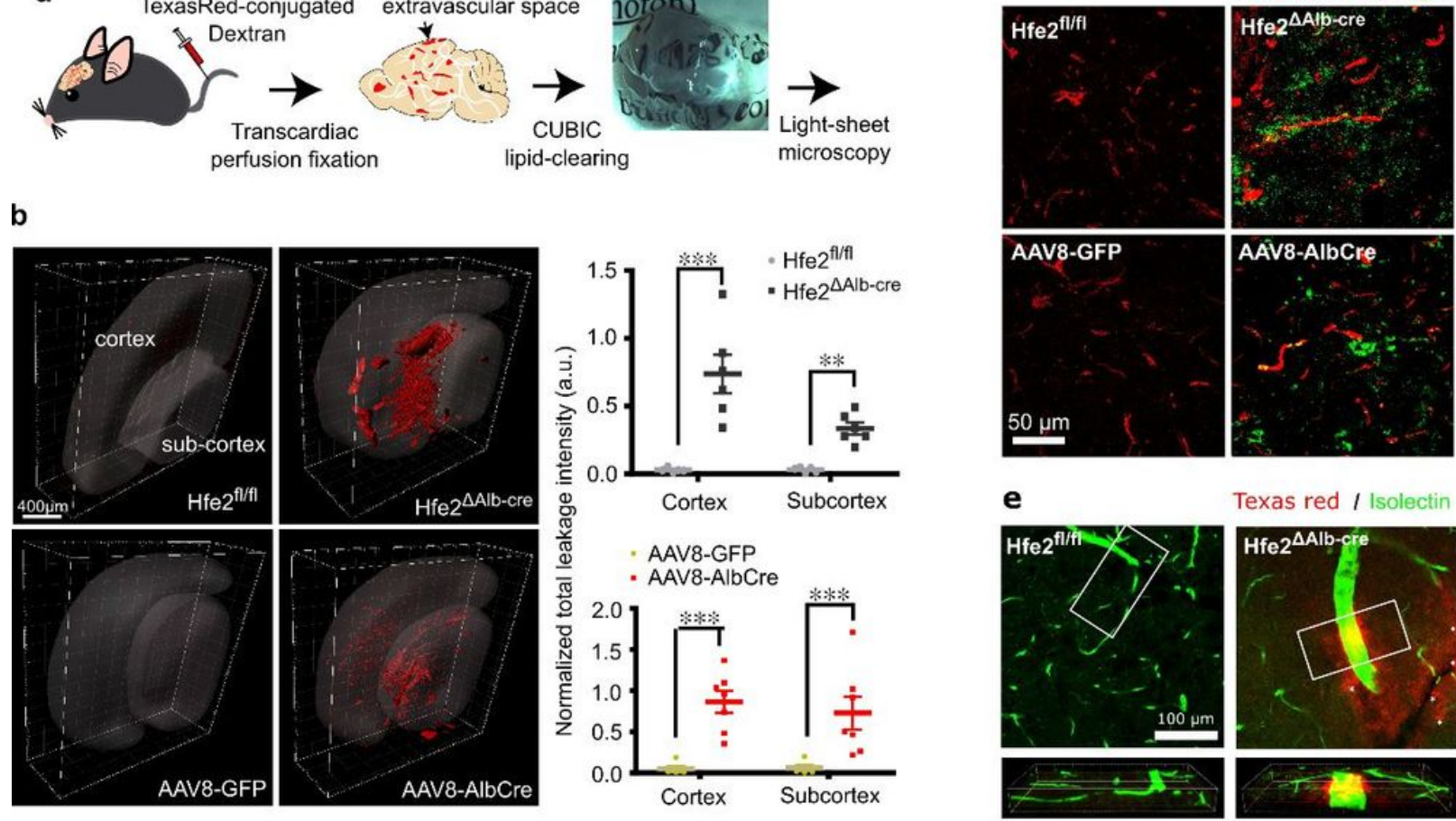

Normalized perivascular fibrinogen intensity (a.u.)
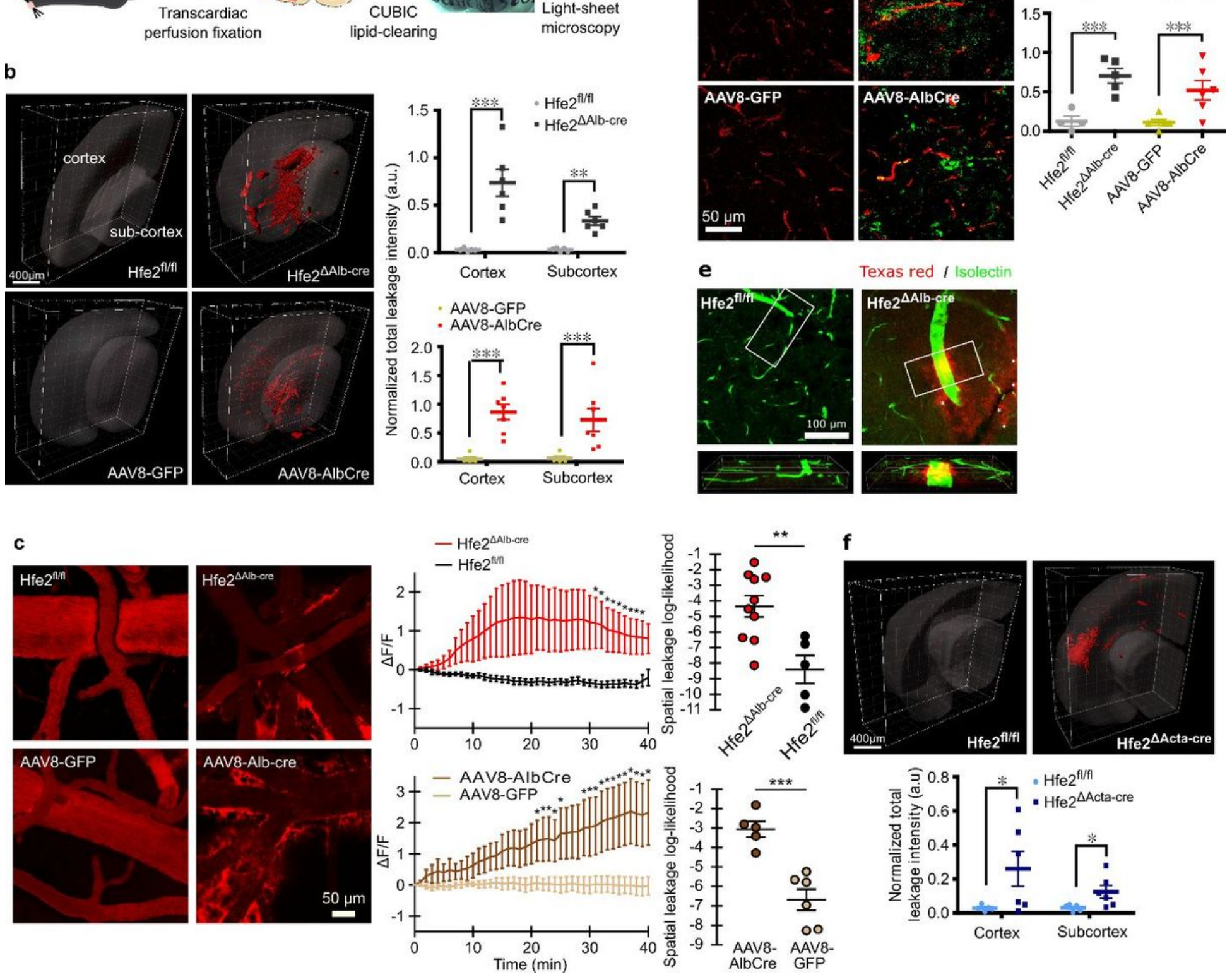

\section{Figure 1}

Hfe2-deficient mice displays severe BBB disruption. a, Schematic of the BBB assessment method using light-sheet microscopy. b, 3D rendered representative light-sheet microscopic images of TR-dextran deposition in CUBIC-cleared brains (scale bars, $400 \mu \mathrm{m}$ ) and time-series quantification (mean \pm s.e.m.; unpaired t-test; $\mathrm{Hfe} 2 \mathrm{fl} / \mathrm{fl}$ (control) $\mathrm{n}=5$, Hfe2 $\triangle$ AlbCre $\mathrm{n}=5$, AAV8-GFP $\mathrm{n}=5$, AAV8-AlbCre $\mathrm{n}=7$ ). c, Representative in-vivo multiphoton images of TR-dextran at 40 mins time-point (scale bar, $50 \mu \mathrm{m}$ ). The normalized extravascular fluorescence $(\Delta F / F)$ intensity was plotted over time and the groups differences were assessed with spatial leakage log-likelihood (mean \pm s.e.m.; unpaired t-tests and repeated measures factorial ANOVA (rendered as approximate unpaired t-test), respectively; $\mathrm{Hfe} 2 \mathrm{fl} / \mathrm{fl} \mathrm{n}=5, \mathrm{Hfe} 2 \Delta \mathrm{AlbCre} \mathrm{n}=10$, AAV8 GFP $n=6$, AAV8 AlbCre $n=5$ ). d, Representative confocal images of fibrinogen disposition around the cerebral vessels (isolectin) of Hfe2-deficient mice (scale bar, $50 \mu \mathrm{m}$ ) and quantification (mean \pm s.e.m.; 
unpaired t-test; Hfe2fl/fl $n=4, H f e 2 \Delta$ AlbCre $n=5$, AAV8-GFP $n=5$, AAV8-AlbCre $n=6$ ). e, Confocal imaging of TR-dextran deposition in cortical brain slices with endothelium counterstain. $\mathrm{f}, 3 \mathrm{D}$ rendered representative light-sheet microscopic images of TR-dextran deposition in CUBIC-cleared brains (scale bars, $400 \mu \mathrm{m}$; mean \pm s.e.m.; unpaired t-test; Hfe2fl/fl $n=5, H f e 2 \triangle$ ActaCre $n=6) .{ }^{*} P<0.05,{ }^{*} P<0.01,{ }^{*} * * P<0.001$.
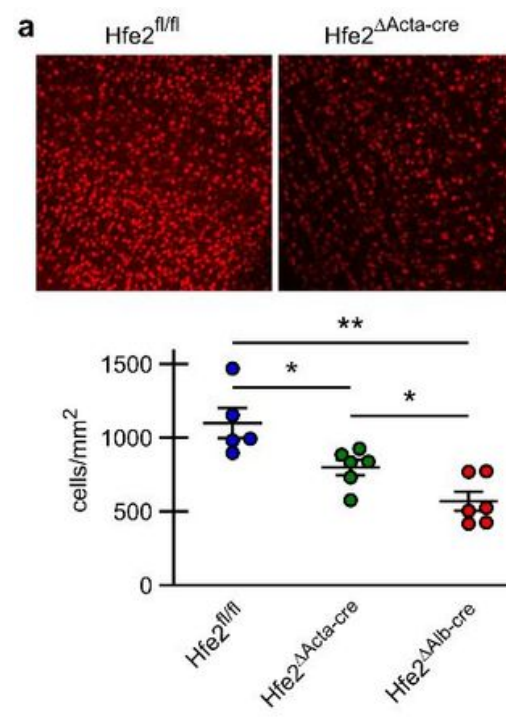

C

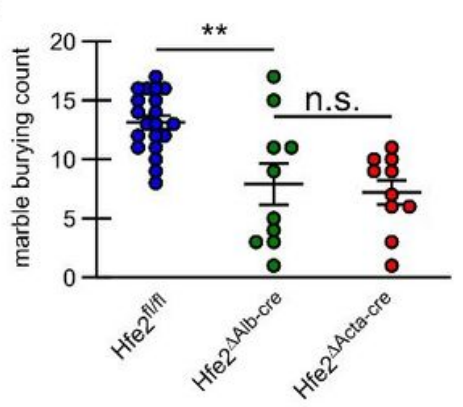

$\mathrm{Hfe} 2^{\Delta \text { Alb-cre }}$
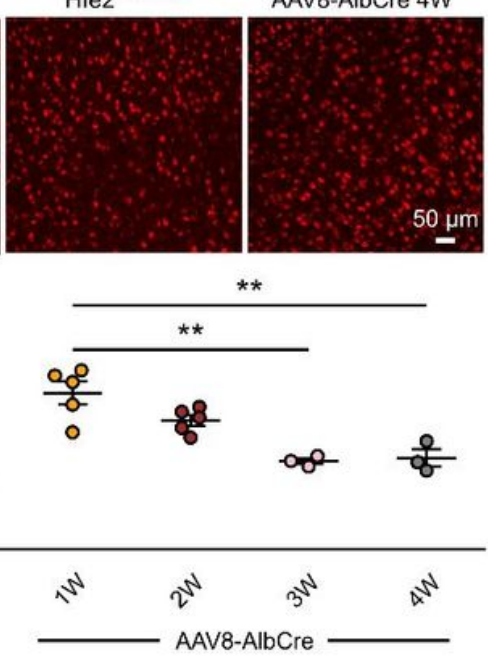

AAV8-AlbCre 4 W

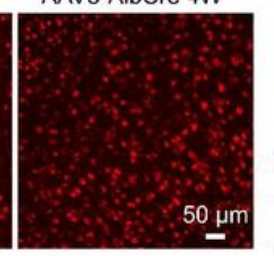

d

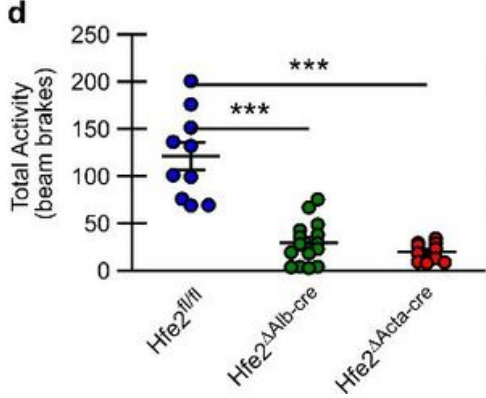

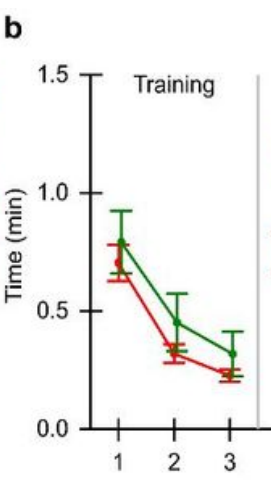

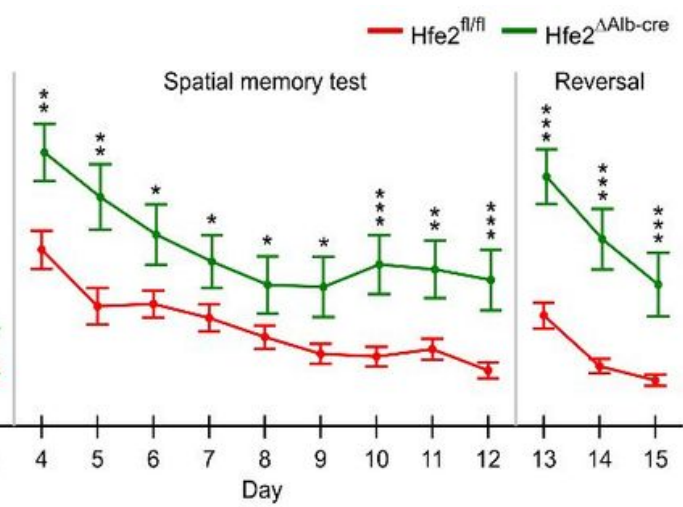

Figure 2
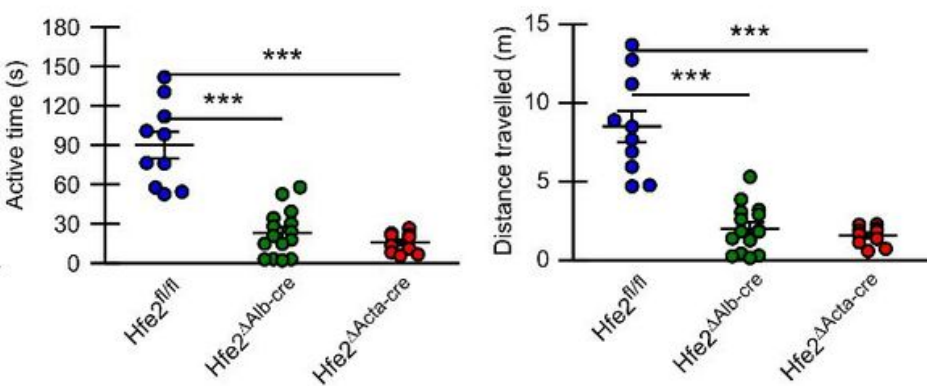

Hfe2 -liver and -muscle knock out affect neuron number and behavior. a, NeuN staining of the same cortical area shows a significant decrease in the number of neurons (scale bar, $50 \mu \mathrm{m}$; mean \pm s.e.m.; unpaired t-tests). All mice were 6-8 weeks old when experiments were initiated. Replicates $\mathrm{Hfe} 2 \mathrm{fl} / \mathrm{fl} \mathrm{n}=6$, Hfe2Alb-cre $n=6$, Hfe2Acta-cre $n=6$, and AAV8-Alb-cre $n=5,5,3$, and 3 at weeks 1, 2, 3, and 4 respectively. b, Hfe2 liver knockout mice display behavioral abnormality. Quantification of escape latency shows greater time response in Liver knock out (mean \pm s.e.m.; unpaired t-tests; $\mathrm{Hfe} 2 \mathrm{fl} / \mathrm{fl} \mathrm{n}=10$ and $\mathrm{Hfe} 2 \Delta \mathrm{Alb}$-cre $\mathrm{n}=5$ ). c, Bar graph of marbles buried by $\mathrm{Hfe} 2 \mathrm{KO}$ mice (mean \pm s.e.m.; unpaired t-tests; $\mathrm{Hfe} 2 \mathrm{fl} / \mathrm{fl} \mathrm{n}=20$, $\mathrm{Hfe} 2 \Delta$ Alb-cre $\mathrm{n}=10$, and Hfe2 $\triangle$ Acta-cre $n=10$ ). d, Bar graph of three main open field test criteria for Hfe2 $\mathrm{KO}$ mice (mean \pm s.e.m.; unpaired t-tests; $\mathrm{Hfe} 2 \mathrm{fl} / \mathrm{fl} \mathrm{n}=10$, Hfe2 $\Delta$ Alb-cre $\mathrm{n}=15$, and Hfe2 $\Delta$ Acta-cre $\mathrm{n}=10$ ). ${ }^{\star} \mathrm{P}<0.05,{ }^{*} \mathrm{P}<0.01, \star \star * * \mathrm{P}<0.001$. 
a

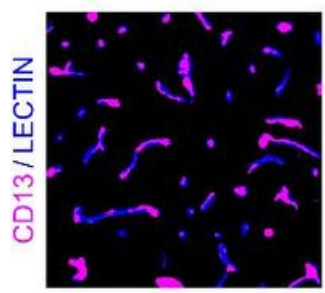

AAV8-GFP
Pericyte coverage

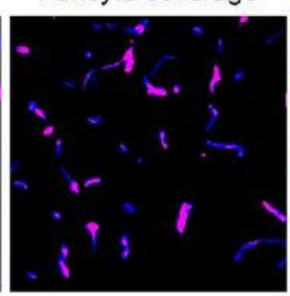

AAV8-alb-cre

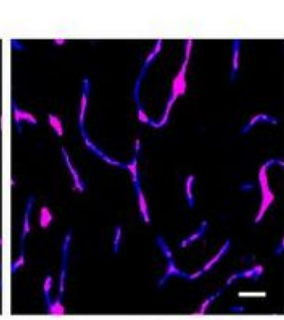

$\mathrm{Hfe}^{\Delta \mathrm{alb}-\mathrm{cre}}$

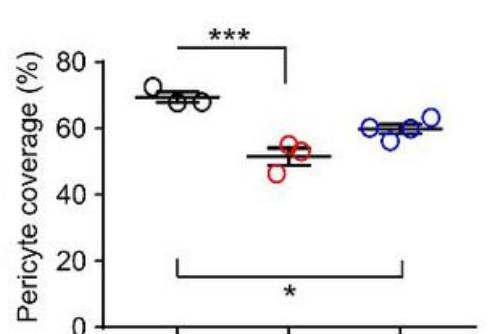

b brain extracted endothelial cells

cultured bEnd. 3 cells
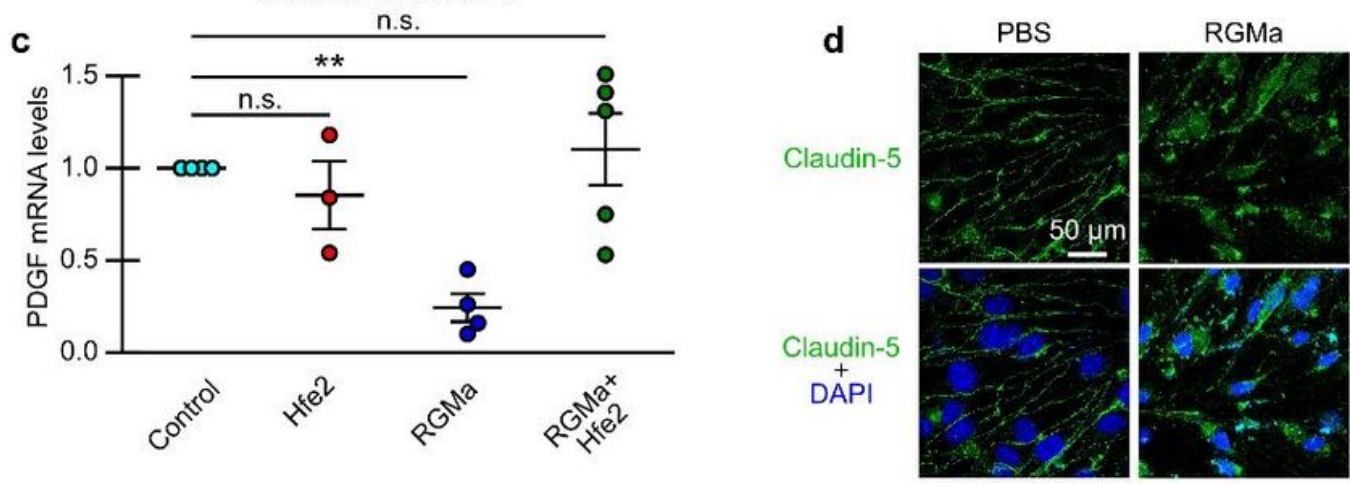

$\mathrm{RGMa}+\mathrm{Hfe} 2$
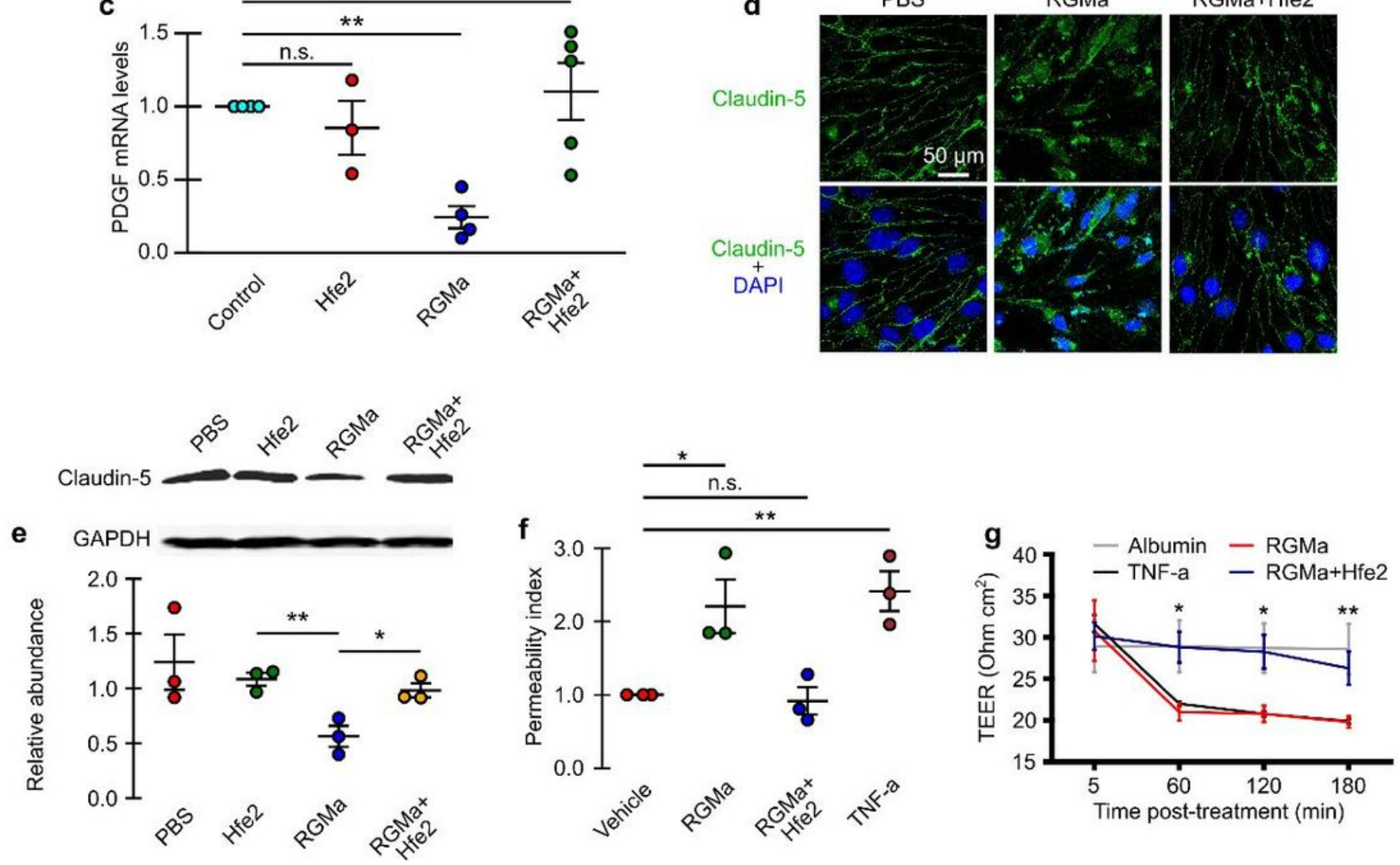

\section{Figure 3}

Soluble Hfe2 and RGMa effects on endothelial cells. a, Knock-out of liver Hfe2 reduces pericyte coverage (mean \pm s.e.m.; unpaired t-tests; AAV8-GFP $n=3, \mathrm{Hfe} 2 \Delta$ Alb-cre $n=4$, and AAV8-AlbCre $n=3$ ). Scale bar, $50 \mu \mathrm{m}$. b, Quantification through quantitative RT-PCR shows a reduction in PDGF-B mRNA levels in liver knock out animals (mean \pm s.e.m.; paired t-test; $\mathrm{Hfe} 2 \mathrm{fl} / \mathrm{fl} \mathrm{n}=3$ and $\mathrm{Hfe} 2 \Delta$ Alb-cre $\mathrm{n}=3$ ). c, Quantification of PDGF-B mRNA in cultured bEnd. 3 cells following indicated treatments. Treatment with RGMa leads to a significant reduction of PDGF-B mRNA levels that is recued with $\mathrm{Hfe} 2$ addition (mean \pm s.e.m.; paired ttests; Hfe2 n=3, RGMa n=4, and RGMa+Hfe2 n=5). d, Immunocytochemistry of claudin-5 in bEnd3 monolayer after $\mathrm{Hfe} 2$ and RGMa treatments. Scale bar, $50 \mu \mathrm{m}$. e, Western blotting of claudin- 5 expression in bEnd 3 cell lysates after indicated treatment (mean \pm s.e.m.; one-way ANOVA with post-hoc Tukey test; replicates $n=3$ ). $f$, Transwell permeability leakage assay performed on a monolayer of bEND3 cells (mean \pm s.e.m.; one-way ANOVA with post-hoc Tukey test; replicates $n=3$ ). $g$, In vitro TEER analysis shows that 
RGMa alters the integrity of a monolayer of bEnd3 cells, this is rescued by Hfe2 (mean \pm s.e.m.; two-way ANOVA with post-hoc Bonferroni test; replicates $n=3)$. ${ }^{*} P<0.05,{ }^{*} \mathrm{P}<0.01,{ }^{*} * \mathrm{P}<0.001$.

a

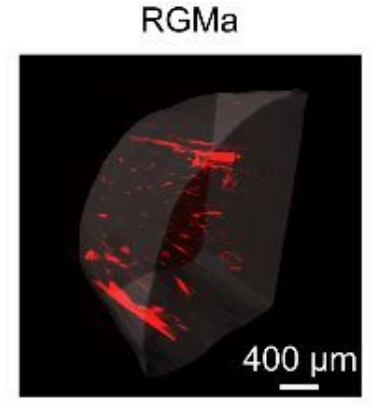

$\mathrm{RGMa}+\mathrm{Hfe} 2$

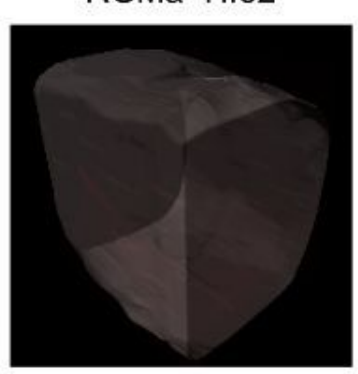

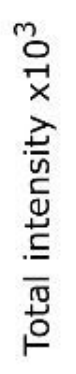

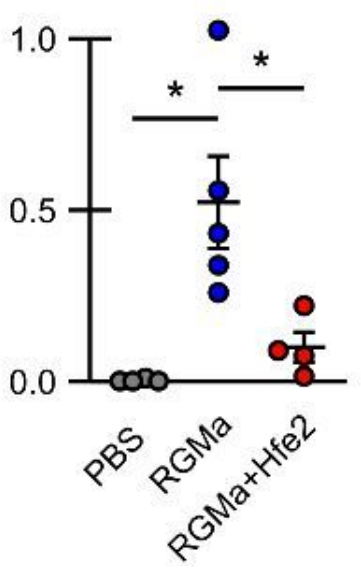

b
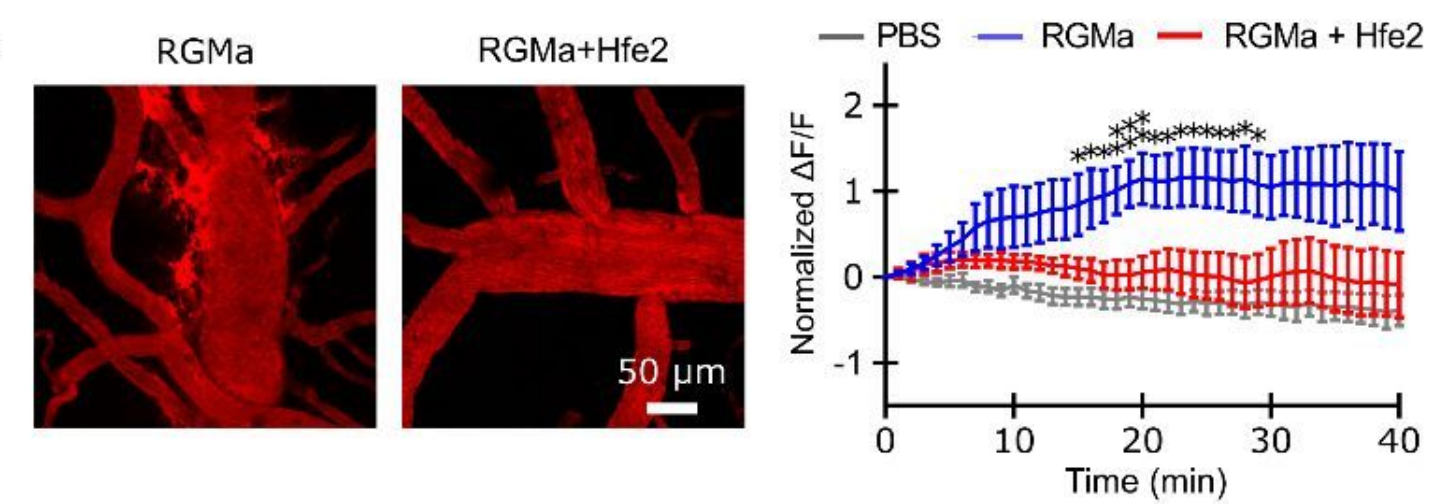
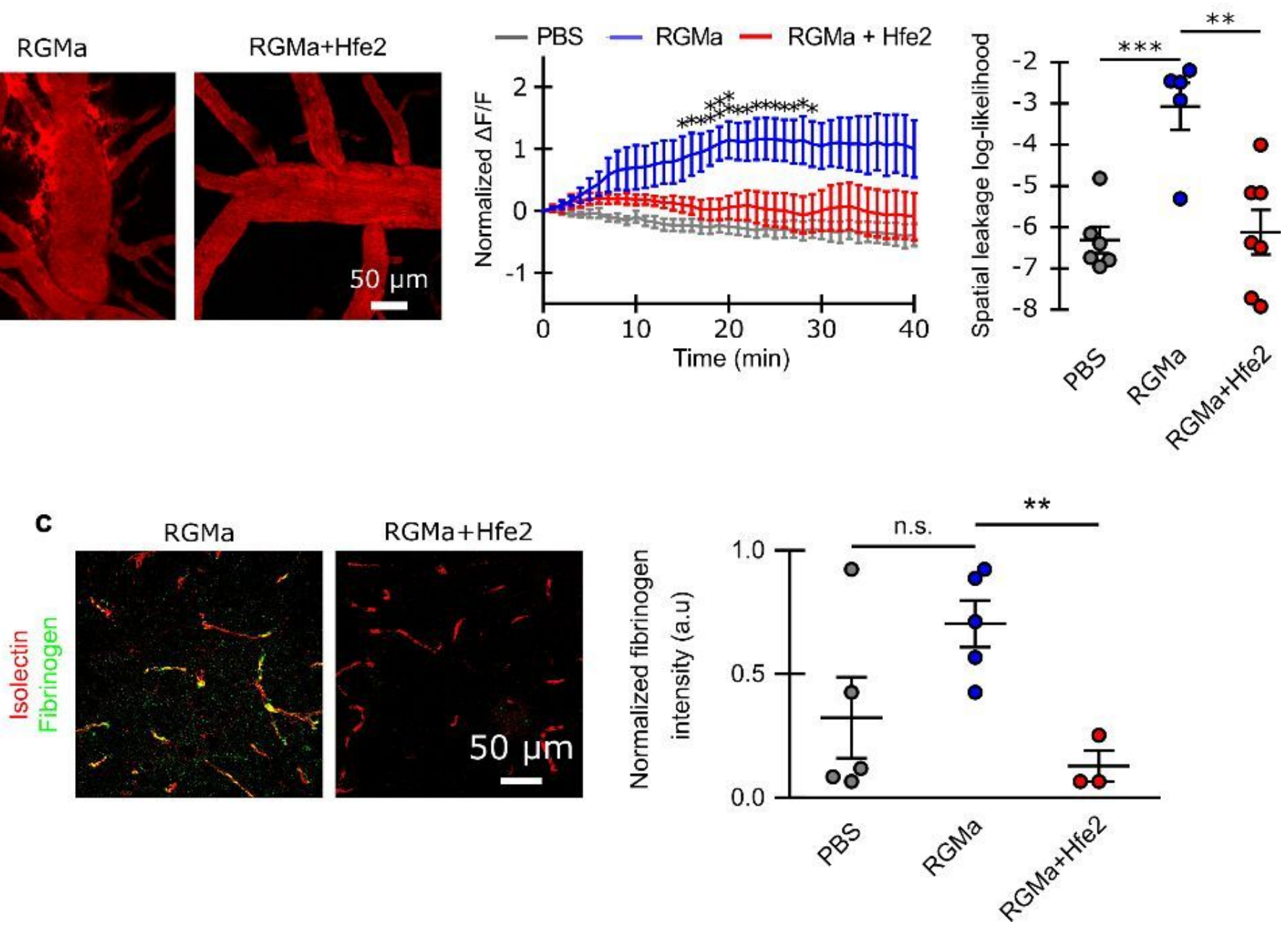

Figure 4

Hfe2 and RGMa have opposite effects on BBB integrity. a, 3D rendered representative light-sheet microscopic images of RGMa and RGMa+Hfe2 treated brains (scale bar, $400 \mu \mathrm{m}$ ) and quantification (mean \pm s.e.m.; unpaired t-tests; PBS n=3, RGMa n=5, RGMa+Hfe2 n=4). b, Representative in-vivo multiphoton images of RGMa and RGMa+Hfe2 treated brains. The normalized extravascular fluorescence $(\Delta F / F)$ intensity was plotted over time and the group differences were assessed with spatial leakage log- 
likelihood (mean \pm s.e.m.; unpaired t-tests and repeated measures factorial ANOVA (rendered as approximate unpaired t-test), respectively; PBS $n=6, R G M a n=5, R G M a+H f e 2 n=7$ ). c, Representative confocal images of fibrinogen disposition around the cerebral vessels (isolectin) of RGMa and $\mathrm{RGMa}+\mathrm{Hfe} 2$ treated mice and quantification (mean \pm s.e.m.; unpaired t-test; PBS $n=5, \mathrm{RGMa} n=5$, $\mathrm{RGMa}+\mathrm{Hfe} 2 \mathrm{n}=3) .{ }^{*} \mathrm{P}<0.05,{ }^{*} \mathrm{P}<0.01, * * * \mathrm{P}<0.001$.
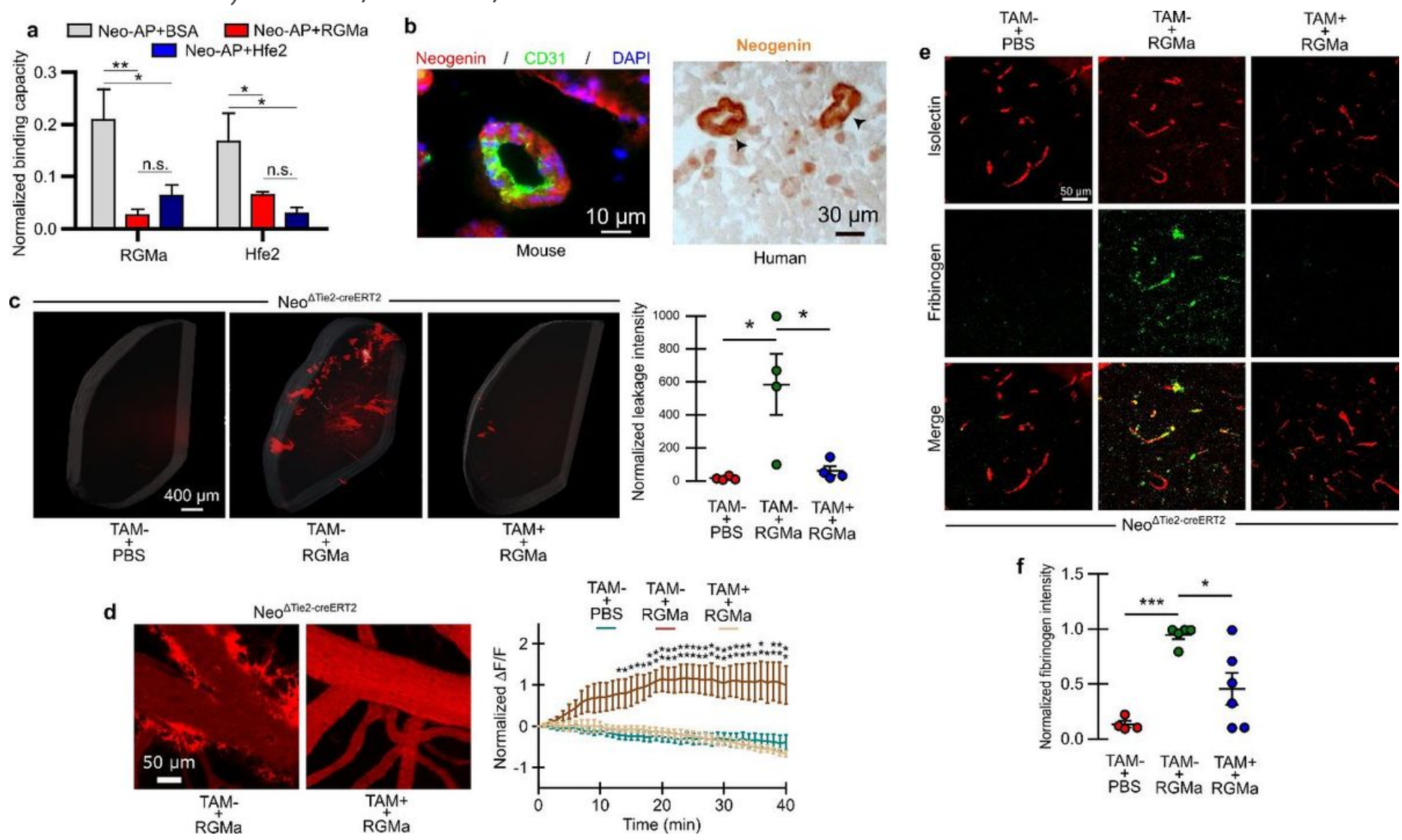

\section{Figure 5}

RGMa disrupts BBB through Neogenin receptor. a, Hfe2 blocks RGMa-interaction with Neogenin. Competitive binding between RGMa and Hfe2 on Neogenin-AP. RGMa blocks binding to Hfe2 and Hfe2 to RGMa. (mean \pm s.e.m.; one-way ANOVA with post-hoc Tukey test; replicates $n=3$ ). b, Expression of Neogenin in the cerebral endothelial cells (CD31) in mice (scale bar, $10 \mu \mathrm{m}$ ) and vascular structure in human (arrows; scale bar, $30 \mu \mathrm{m}$ ). c, Representative 3D light-sheet images of Neo $\Delta$ Tie2-creERT mice treated (TAM+) and untreated (TAM-) with Tamoxifen before subsequent RGMa treatment (scale bars, $400 \mu \mathrm{m}$ ) and quantification (mean \pm s.e.m.; unpaired t-test; TAM- \& PBS $n=4, T A M-\&$ RGMa $n=4, T A M+\&$ RGMa $\mathrm{n}=4$ ). d, Representative in-vivo multiphoton images at 45 mins time-point (scale bar, $50 \mu \mathrm{m}$ ) and time-series quantification (mean \pm s.e.m.; two-way ANOVA with post-hoc Bonferroni test; TAM- \& PBS $n=4$, $T A M-\& R G M a n=4, T A M+\& R G M a n=4)$. e-f, Representative confocal images of fibrinogen staining in the brain (e; scale bar, $50 \mu \mathrm{m}$ ) and quantification (f; mean \pm s.e.m.; unpaired t-test; TAM- \& PBS $n=4$, TAM- \& RGMa $n=5, T A M+\& R G M a n=6) .{ }^{*} P<0.05, * * P<0.01, * \star * P<0.001$. 

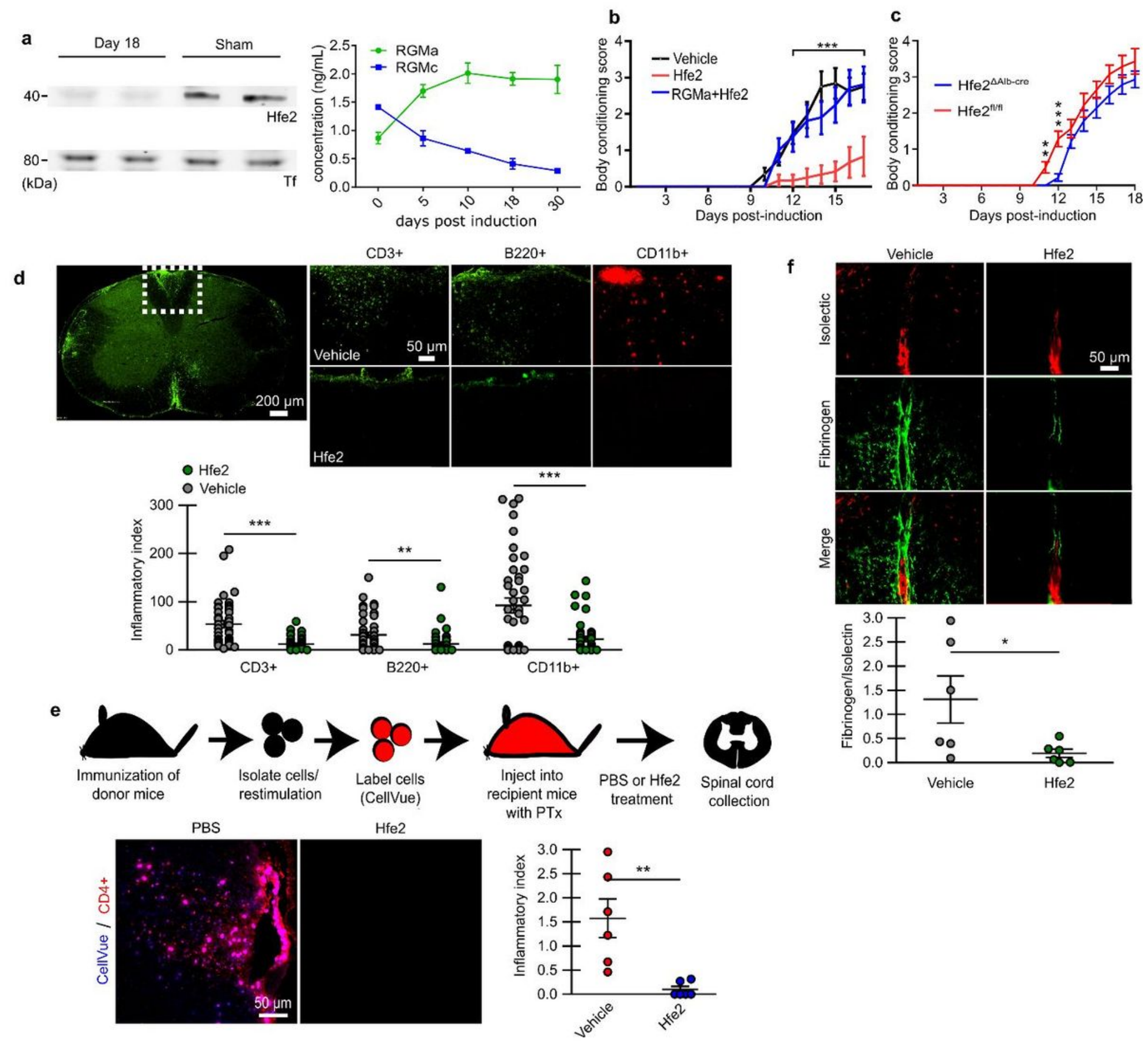

\section{Figure 6}

Hfe2 administration significantly improves EAE disease outcome. a, Western Blot analysis shows a strong $\mathrm{Hfe} 2$ reduction in EAE animal serum when compared to control. ELISA quantification showing $\mathrm{Hfe} 2$ decrease and RGMa increase in EAE animal serum (mean \pm s.d.; EAE $n=3$ and RGMa $n=3$ ). b, Body conditioning assessment (' 0 ' no paralysis to '4' forelimb weakness) of EAE-induced mice with Hfe2+/RGMa treatment every 3 days (d3, 6, 9, 12 and 15; mean \pm s.e.m.; unpaired t-tests; PBS $n=8, H f e 2 n=6$, RGMa+Hfe2 $\mathrm{n=8}$ ). c, Body conditioning assessment of EAE-induced Hfe2fl/fl and Hfe2 $\Delta$ Alb-cre mice (mean \pm s.e.m.; unpaired t-tests; Hfe2fl/fl $n=8$ and Hfe2 $\Delta$ Alb-cre $n=8$ ). d, Representative confocal images of CD3+, B220+ and CD11b+ immune staining in spinal cord of EAE-induced mice 3 weeks after induction and quantification (mean \pm s.e.m.; unpaired t-test; PBS n=7, Hfe2 $n=6$ ). e, Schematic of activated immune 
cell adoptive transfer. Representative confocal images of CD4-positive labelled transferred cell (CellVue) in PBS-treated or Hfe2-treated recipient WT mice and quantification (mean \pm s.e.m.; unpaired t-test; PBS $n=6, \mathrm{Hfe} 2 \mathrm{n=6}$ ). $\mathrm{f}$, Representative confocal images of fibrinogen in the spinal cord of Hfe2-treated EAEmice and quantification (mean \pm s.e.m.; unpaired t-test; PBS-treated $n=6$ and $\mathrm{Hfe} 2 \mathrm{n}=6$ ). ${ }^{*} P<0.05$, $\star \star P<0.01, * \star * P<0.001$.

\section{Supplementary Files}

This is a list of supplementary files associated with this preprint. Click to download.

- Methodsv12naturecardiovascularresearch.docx

- Supplementaryfiguresv10naturecardiovascularresearch.docx

- Movie1.avi

- Movie2.avi

- Movie3.avi

- Movie4.avi 